\title{
Roles of tRNA metabolism in aging and lifespan
}

\author{
Zheng Zhou', Bao Sun ${ }^{2,3}$, Dongsheng Yu (i) and Meng Bian (1D)
}

\begin{abstract}
Transfer RNAs (tRNAs) mainly function as adapter molecules that decode messenger RNAs (mRNAs) during protein translation by delivering amino acids to the ribosome. Traditionally, tRNAs are considered as housekeepers without additional functions. Nevertheless, it has become apparent from biological research that tRNAs are involved in various physiological and pathological processes. Aging is a form of gradual decline in physiological function that ultimately leads to increased vulnerability to multiple chronic diseases and death. Interestingly, tRNA metabolism is closely associated with aging and lifespan. In this review, we summarize the emerging roles of tRNA-associated metabolism, such as tRNA transcription, tRNA molecules, tRNA modifications, tRNA aminoacylation, and tRNA derivatives, in aging and lifespan, aiming to provide new ideas for developing therapeutics and ultimately extending lifespan in humans.
\end{abstract}

\section{Facts}

1. tRNAs are important participants in protein translation and are involved in various physiological and pathological processes.

2. tRNA-associated metabolism is closely associated with aging and lifespan.

3. The enzymes related to tRNA metabolism could be potential targets for future therapeutic interventions in aging and lifespan.

\section{Open questions}

1. Is tRNA metabolism involved in the regulation of aging and lifespan mainly by affecting protein synthesis?

2. What is the molecular mechanism by which tRNA derivatives regulate aging and lifespan?

3. Is there potential for practical clinical applications

Correspondence: Dongsheng Yu (dongshyu@163.com) or

Meng Bian (bianmeng0208@163.com)

'Department of Chinese Medicine, The First Affiliated Hospital of Zhengzhou University, Zhengzhou 450000, China

${ }^{2}$ Department of Pharmacy, The Second Xiangya Hospital, Central South University, Changsha 410011, China

Full list of author information is available at the end of the article

These authors contributed equally: Zheng Zhou, Bao Sun

Edited by B. Zhivotovsky based on findings concerning tRNAs in the context of aging and lifespan?

\section{Introduction}

Transfer RNAs (tRNAs) are important participants in protein translation, which transport their cognate amino acids to the ribosome. There are more than 600 putative tRNA genes in the human genome, some of which are transcribed into precursor tRNAs (pre-tRNAs) by RNA polymerase III (Pol III $)^{1}$. Subsequently, pre-tRNAs are transformed into mature tRNAs after a series of processing and modification processes, which are characterized by a "clover" secondary structure as well as an L-shaped tertiary structure ${ }^{2}$. After maturation, tRNAs are charged with their cognate amino acids through the aminoacylation reactions mediated by aminoacyl-tRNA synthetases (ARSs), thereby participating in protein translation ${ }^{3}$. Of note, tRNAs will be cleaved into fragments with regulatory functions under stress conditions ${ }^{4-6}$. In general, normal tRNA metabolism is essential to maintain the stability and functions of tRNA molecules, while the defects in certain tRNA biogenesis proteins cause various human diseases, including cancer, neurological disorders, immunodeficiency, and diabetes mellitus ${ }^{7-10}$.

Aging is a complex physiological process, usually manifested by a gradual decline in organ function, as well as an increase in disease incidence and risk of death. It is reported that the global population over 65 will reach 1.6

\section{(c) The Author(s) 2021}

(c) Open Access This article is licensed under a Creative Commons Attribution 4.0 International License, which permits use, sharing, adaptation, distribution and reproduction cc) in any medium or format, as long as you give appropriate credit to the original author(s) and the source, provide a link to the Creative Commons license, and indicate if changes were made. The images or other third party material in this article are included in the article's Creative Commons license, unless indicated otherwise in a credit line to the material. If material is not included in the article's Creative Commons license and your intended use is not permitted by statutory regulation or exceeds the permitted use, you will need to obtain permission directly from the copyright holder. To view a copy of this license, visit http://creativecommons.org/licenses/by/4.0/. 
billion by $2050^{11}$. In fact, delaying biological aging or extending healthspan is an eternal theme of human health ${ }^{12,13}$. Strikingly, tRNAs play an important role in aging and lifespan. For example, the serum levels of mitochondrial tRNAs and ribosomal RNAs (rRNAs) will increase with age, which may be related to mitochondrial dysfunction during the aging process ${ }^{14}$. Another research discovered that Pol III was a downstream molecule of Target of Rapamycin Complex 1 (TORC1), and its inhibition could extend organismal lifespan ${ }^{15}$. Moreover, the deletion of nucleoporin Nup100 could regulate the life span of Saccharomyces cerevisiae by inhibiting the nuclear export of specific tRNAs ${ }^{16}$. Therefore, tRNAs are closely related to aging biology and thus participate in the regulation of age-related diseases and lifespan. Here, we focus on the roles of tRNA-associated metabolism, such as tRNA transcription, tRNA molecules, tRNA modifications, tRNA aminoacylation, and tRNA derivatives, in aging and lifespan, which may serve as novel targets for lifespan extension (Fig. 1).

\section{Roles of tRNA metabolism in aging and lifespan tRNA transcription in aging and lifespan}

Small ubiquitin-related modifier modification (SUMOylation) is a highly dynamic post-translational modification that has been confirmed to be related to transcriptional repression ${ }^{17,18}$. Meanwhile, many studies have linked SUMOylation to aging process ${ }^{19,20}$. In eukaryotes, three essential RNA polymerases are evolutionarily conserved enzymes responsible for the transcription of their nuclear genomes. Of these, Pol I mainly transcribes the 25S rRNA precursor, Pol II transcribes various messenger RNAs (mRNAs), while Pol III transcribes short RNAs such as tRNAs and $5 \mathrm{~S}$ rRNA $^{21,22}$. Interestingly, SUMO machinery was widely distributed in the genome, especially at the promoters of histone and protein biogenesis genes, as well as Pol I-transcribed rRNA genes and Pol III-transcribed tRNA genes ${ }^{23}$. Surprisingly, the SUMO machinery was selectively retained on histone and tRNA genes and released in large quantities from other chromatin in senescent cells, indicating that maintaining the suppression of histone and tRNA loci was beneficial to the stability of the aging state ${ }^{23}$. These data support that SUMOylation-mediated coordinated repression of a transcriptional program is associated with cell growth and proliferation.

It is well-known that TORC1 is an important longevity determinant among many species ${ }^{24-26}$. Recent studies observed that the GATA transcription factor Gaf1 deficiency could shorten the normal chronological lifespan and reduce the lifespan extension caused by TORC1 inhibition in yeast ${ }^{27}$. Specifically, upon TORC1 block, Gaf1 served as a transcription factor downstream of TORC1 that directly bound to Pol III-transcribed tRNA genes and inhibited their transcription, thereby promoting longevity by inhibiting translation. Strikingly, Pol III mediated the longevity-promoting effects of TORC1 inhibition ${ }^{15}$. In this condition, systemic Pol III deficiency could facilitate the longevity in yeast, flies, and worms, and gut-specific inhibition of Pol III in adult worms or flies was sufficient to prolong the lifespan, which might be related to the reduced protein synthesis and increased resistance to proteotoxic stress. Importantly, the effects of Pol III inhibition and rapamycin treatment on lifespan extension were not additive ${ }^{15}$. Rapamycin treatment suppressed the phosphorylation of TORC1 substrate in

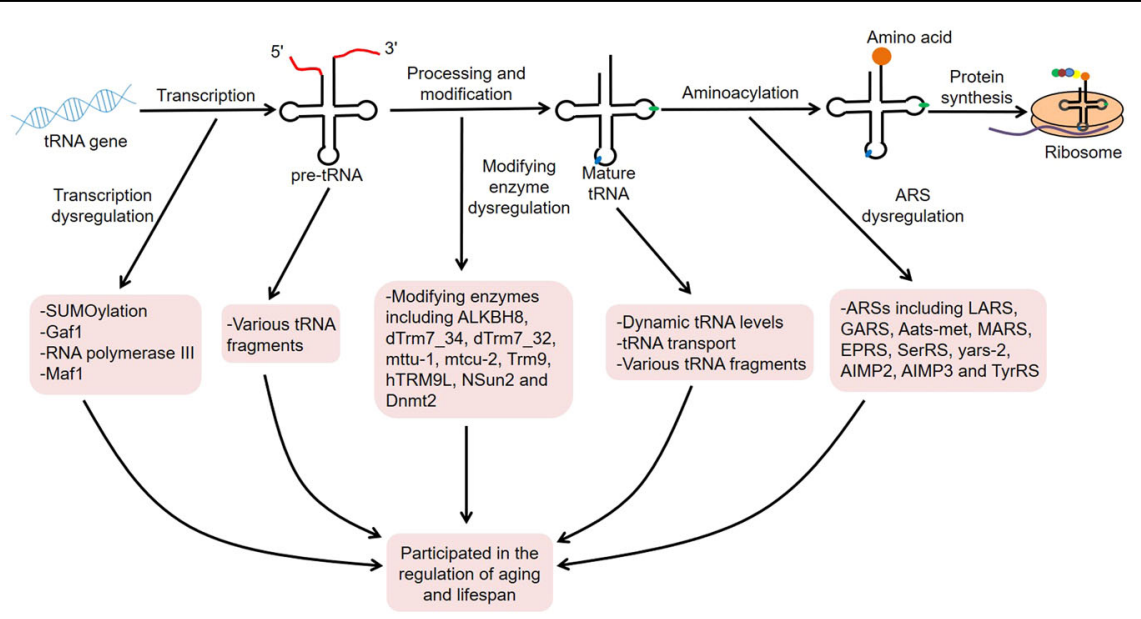

Fig. 1 Roles of tRNA-associated metabolism in aging and lifespan. The tRNA genes are transcribed into pre-tRNAs, which are then transformed into mature tRNAs after a series of processing and modifications. Subsequently, mature tRNAs are involved in protein translation. During this process, tRNA-associated metabolism, such as tRNA transcription, tRNA molecules, tRNA modifications, tRNA aminoacylation, and tRNA derivatives, plays an important role in aging and lifespan. 
the intestine, while the gut-specific Pol III inhibition did not, indicating that Pol III acted as a downstream molecule of TORC1 to regulate lifespan.

TORC1 directly phosphorylated MAF1 homolog negative regulator of Poll III (Maf1) at multiple sites and thus controlled its localization and Pol III-mediated transcription ${ }^{28-30}$. Notably, inhibition of TORC1 by rapamycin treatment reduced pre-tRNA levels in whole flies, and overexpression of gut-specific Maf1 reduced pre-tRNA levels and prolonged lifespan, indicating that Maf1mediated Pol III inhibition might be involved in the regulation of lifespan by mTOR pathway ${ }^{15}$. Moreover, mafi $1 \Delta$ cells showed a shorter lifespan under lower glucose conditions, and the short lifespan was rescued by introducing the plasmid encoding maf1 gene, which suggested that Maf1 was required for lifespan extension of Schizosaccharomyces pombe ${ }^{31}$. Maf1 was phosphorylated by TORC1 under high-calorie conditions, while it was dephosphorylated by PP2A and PP4 under calorierestricted conditions. The phosphorylation status of Maf1 was associated with $S$. pombe lifespan and tRNA levels. Importantly, Maf1-dependent inhibition of tRNA transcription extended lifespan in fission yeast mainly by preventing genomic instability at tRNA genes, rather than inhibiting protein synthesis ${ }^{31}$. Further studies discovered that the break of tRNA genes was caused by replicationtranscription conflicts, while Maf1 could limit Pol IIImediated transcription to maintain genomic integrity ${ }^{32}$. These findings indicate that transcription-related genomic instability may play an important role in the aging process. Intriguingly, Maf1 deletion increased the lifespan in worms and mice, which also indicated that Maf1 participated in lifespan regulation through complex mechanisms, not just by regulating Pol III output ${ }^{33,34}$.

\section{tRNA molecules in aging and lifespan}

In addition, tRNA molecules are also involved in the regulation of aging and lifespan. Sagi et al. ${ }^{35}$ demonstrated that tRNA expression decreased with age in worms, and the higher sup- 7 tRNA levels at day 6 were associated with a longer lifespan. The decline in tRNA expression might cause protein misfolding, leading to the development of age-related diseases. Moreover, nuclear tRNA accumulation was related to the increased replicative lifespan in yeast $^{36}$. In this context, deletion of tRNA exporter Los1 could significantly extend lifespan. Mechanistically, dietary restriction excluded Los1 from the nucleus in a manner dependent on Rad53 and mTOR, thereby promoting nuclear tRNA accumulation and transcription factor Gcn4 activation. Analogously, deletion of Nup100 facilitated the expression of Gcn4 by suppressing the nuclear export of tRNAs and thus contributed to the increased longevity in S. cerevisiae ${ }^{16}$. nup $100 \Delta$ cells did not show tRNA splicing and aminoacylation defects, indicating that Nup100 was mainly responsible for the reexport of several mature tRNAs, such as tRNA ${ }^{\mathrm{Tyr}}$, tRNA $^{\text {Trp }}$, and tRNA ${ }^{\text {Ile }}$. Of note, the localization of Los1 and Msn5 (another protein involved in tRNA export) was not regulated by Nup100, which supported that Nup100 could regulate tRNA export in a manner distinct from them ${ }^{16}$. Together, the dysregulation of tRNA levels and transport may affect the lifespan of organisms.

\section{tRNA modifications in aging and lifespan}

tRNAs always undergo a variety of post-transcriptional modifications, which affect tRNA stability, codon recognition, and even aminoacylation ${ }^{37}$. Strikingly, many studies have demonstrated that certain tRNA-modifying enzymes are involved in the regulation of cellular senescence and lifespan (Table 1). Alkylation repair homolog 8 (ALKBH8) is a tRNA methyltransferase involved in the formation of 5-methoxycarbonylmethyluridine $\left(\mathrm{mcm}^{5} \mathrm{U}\right)$, 5-methoxycarbonylmethyl-2'-O-methyluridine $\left(\mathrm{mcm}^{5} \mathrm{Um}\right), \quad 5$ methoxycarbonylmethyl-2-thiouridine $\left(\mathrm{mcm}^{5} \mathrm{~s}^{2} \mathrm{U}\right)$, and 5methoxycarbonylhydroxymethyluridine $\left(\mathrm{mchm}^{5} \mathrm{U}\right)$ at the anticodon wobble position of tRNAs ${ }^{38-40}$. ALKBH8deficient mouse embryonic fibroblasts showed selenoprotein loss as well as a senescence phenotype characterized by increased levels of senescence-associated $\beta$-galactosidase (SA- $\beta$-Gal), heterochromatin foci, p16 ${ }^{\mathrm{INK} 4 \mathrm{a}}$, and senescence associated secretory phenotype markers $^{41}$. Another research found that dTrm7_34 and dTrm7_32, as functional orthologs of yeast TRM7 and human FtsJ RNA 2'-O-methyltransferase 1 (FTSJ1), catalyzed 2'-O-Methylation (Nm) at specific tRNAs in Drosophila ${ }^{42}$. Interestingly, $\mathrm{Nm}$ at position $\mathrm{G}_{34}$ limited the cleavage of tRNA ${ }^{\text {Phe }}$, while the $3^{\prime}$ terminal $\mathrm{Cm}_{32}$ might stabilize the $\mathrm{TRNA}^{\text {Phe }}$ fragments that were produced in dTrm7_34 mutants. Meanwhile, the mutant animals of dTrm7_34 and dTrm7_32 exhibited small RNA pathway dysfunctions, increased susceptibility to RNA virus infection, and shortened lifespan, suggesting that these two methyltransferases appeared to modulate the small RNA silencing and lifespan in adult flies ${ }^{42}$.

In mammals, mitochondrial translation optimization factor 1 (MTO1) catalyzed the formation of $\mathrm{tm}^{5} \mathrm{U}$ at anticodon position 34 in certain mitochondrial tRNAs (mt-tRNAs) ${ }^{43}$. The loss of MTO1 affected translation fidelity through defective tRNA modification in mice, resulting in tissue-specific oxidative phosphorylation (OXPHOS) defects. mttu-1 and mtcu-2 in Caenorhabditis elegans were the homologs of tRNA 5methylaminomethyl-2-thiouridylate methyltransferase (TRMU) and $\mathrm{MTO1}^{44}$ (Fig. 2a). Notably, the lifespan of mttu-1 mutants was slightly extended at $20^{\circ} \mathrm{C}$, and that of mtcu- 2 and mttu-1 double mutants was significantly extended, which was associated with the OXPHOS dysfunction in C. elegans. These findings indicate that these 


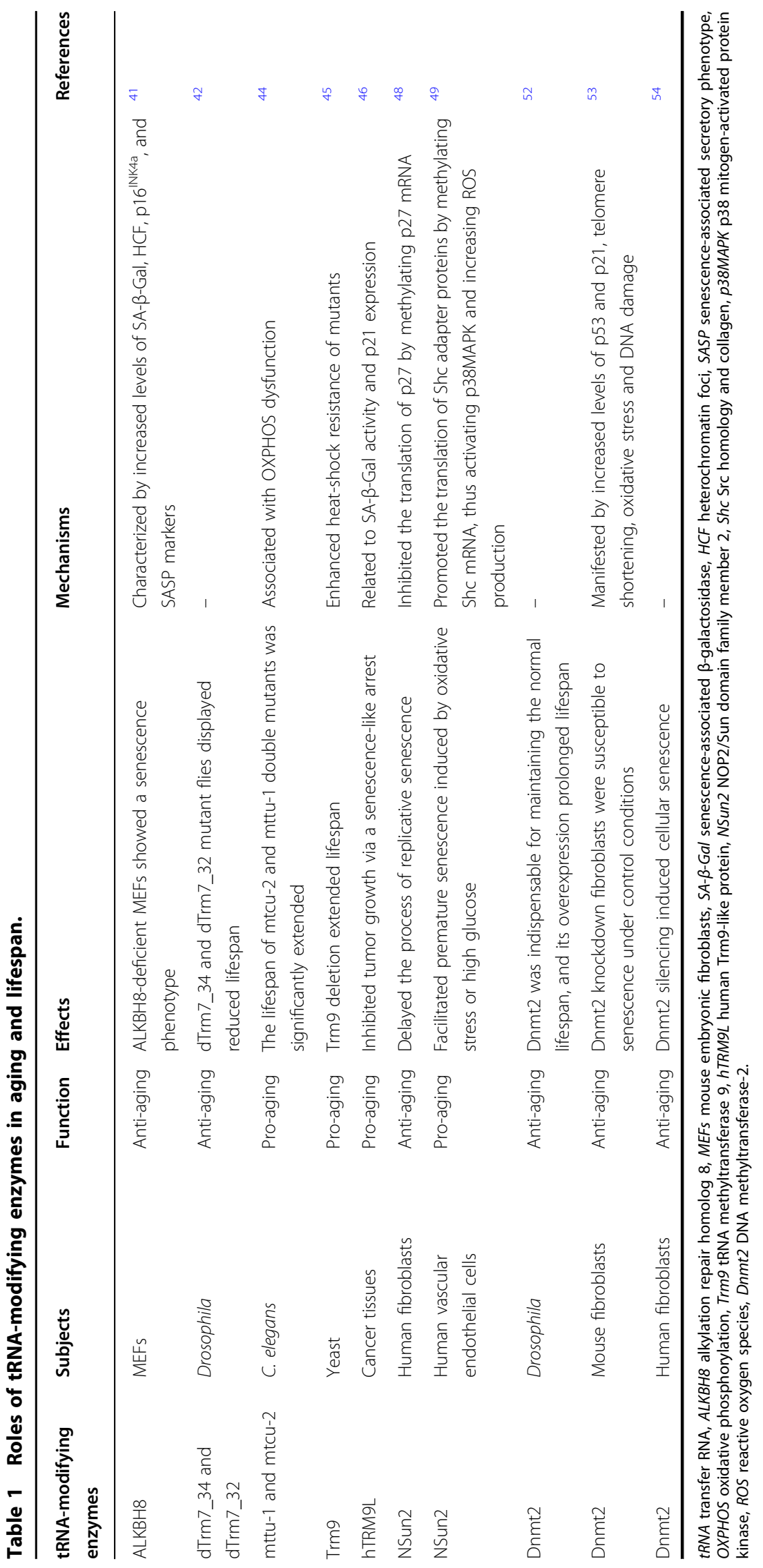




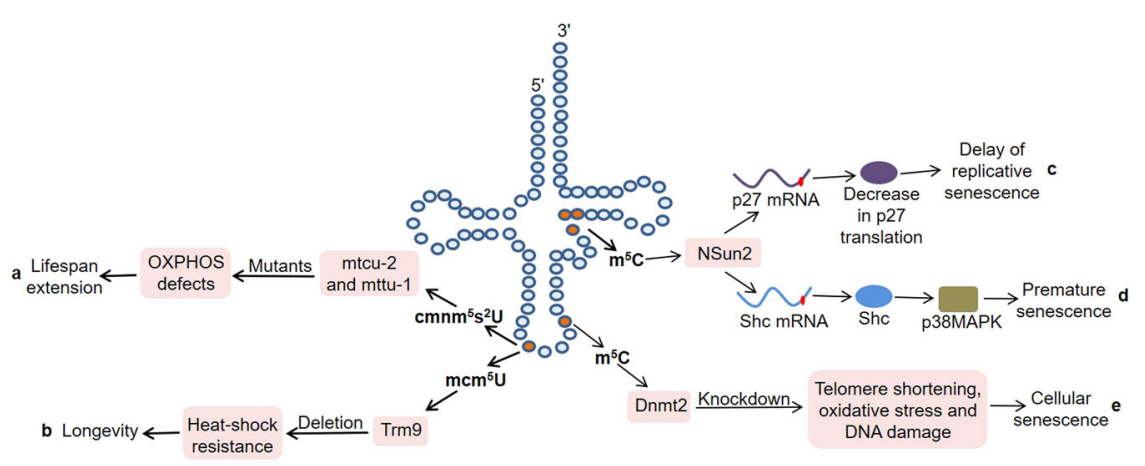

Fig. 2 The dysregulation of tRNA-modifying enzymes in aging and lifespan. a The mtcu-2 and mttu-1 mutants show OXPHOS dysfunction, resulting in lifespan extension. $\mathbf{b}$ Trm9 deletion enhances heat-shock resistance of mutants, thereby supporting the link between longevity and cellular protection. c NSun2 inhibits the translation of p27 by methylating p27 mRNA, thereby delaying the process of replicative senescence. $\mathbf{d}$ NSun2 promotes the translation of Shc adapter proteins by methylating Shc mRNA. Subsequently, the increased Shc proteins activate p38MAPK, thereby facilitating premature senescence. e Dnmt2 knockdown fibroblasts are susceptible to senescence under control conditions, manifested by telomere shortening, oxidative stress, and DNA damage.

two modifying enzymes may be synergistic in regulating the lifespan of worms. But the underlying molecular mechanism requires further research. Fabrizio et al. ${ }^{45}$ discovered that the deletion of acyl-CoA binding protein (Acb1), tRNA methyltransferase 9 (Trm9) and CKA2 could significantly extend lifespan by performing a screen of a yeast homozygous deletion collection. Among them, Trm9 was responsible for the formation of 5methoxycarbonyl-methyluridine $\left(\mathrm{mcm}^{5} \mathrm{U}\right)$ at position 34 in tRNA $^{\text {Glu }}$ and tRNA ${ }^{\mathrm{Arg} 3}$. Importantly, their deletion enhanced the heat-shock resistance of mutants, thereby supporting the link between longevity and cellular protection (Fig. 2b). Furthermore, human Trm9-like protein (hTRM9L) was down-regulated in a variety of cancer tissues, and its re-expression significantly inhibited tumor growth in vivo ${ }^{46}$. hTRM9L induced a senescence-like phenotype related to SA- $\beta$-Gal activity and p21 expression. Meanwhile, hTRM9L could upregulate LIN9 and inhibit the hypoxic response, thereby exerting antitumor activity.

Mammalian NOP2/Sun domain family member 2 (NSun2) is responsible for the cytosine-5 methylation $\left(\mathrm{m}^{5} \mathrm{C}\right)$ in specific tRNA molecules, such as tRNA ${ }^{\mathrm{Gly}}$, tRNA $^{\text {Asp }}$, and tRNA ${ }^{\text {Val47 }}$. Interestingly, NSun2 inhibited the translation of p27 by methylating the $5^{\prime}$-untranslated region (UTR) of p27 mRNA, thereby delaying the process of replicative senescence ${ }^{48}$ (Fig. 2c). At the same time, overexpression of the p27 5'UTR fragment could rescue the decrease of p27 and the increase of cyclin-dependent kinase 1 caused by NSun2 overexpression in 2BS cells, indicating that NSun2-mediated mRNA methylation played an important role in replicative senescence ${ }^{48}$. Cai et al. ${ }^{49}$ demonstrated that NSun2 promoted the translation of Src homology and collagen (Shc) adapter proteins, p66SHC, p52SHC, and p46SHC, by methylating She
mRNA at multiple sites (Fig. 2d). Subsequently, the increased Shc proteins activated p38 mitogen-activated protein kinase (p38MAPK) and increased cellular reactive oxygen species production, thereby facilitating the premature senescence of human vascular endothelial cells induced by oxidative stress or high glucose.

In addition, DNA methyltransferase-2 (Dnmt2) specifically methylated cytosine 38 in the anticodon loops of tRNAs ${ }^{50,51}$. It has been confirmed that Dnmt2 is indispensable for maintaining the normal lifespan of Drosophila, and its overexpression can prolong lifespan ${ }^{52}$. Moreover, Dnmt2 was also related to the conditiondependent apoptosis and senescence in mouse fibroblasts $^{53}$. On the one hand, Dnmt2 knockdown fibroblasts were more prone to apoptosis under the stimulation of hydrogen peroxide. On the other hand, these cells were more susceptible to senescence under control conditions, manifested by increased levels of p53 and p21, telomere shortening, oxidative stress, and DNA damage (Fig. 2e). Consistently, Dnmt2 silencing inhibited the proliferation of human fibroblasts and induced cellular senescence ${ }^{54}$. These findings indicate that Dnmt2 may serve as a novel regulator of longevity.

\section{tRNA aminoacylation in aging and lifespan}

It is well known that tRNAs bind to their homologous amino acids through ARS-mediated aminoacylation, thereby transporting amino acids to the ribosome to participate in protein synthesis. In mammalian cells, one part of ARSs exists in free form, while the other part interacts with three ARS-interacting multi-functional proteins (AIMPs) to form a multi-tRNA synthetase complex $(\mathrm{MSC})^{55}$. Intriguingly, ARSs and AIMPs are closely associated with aging and lifespan (Table 2). Previous studies found that a null mutation in mitochondrial 


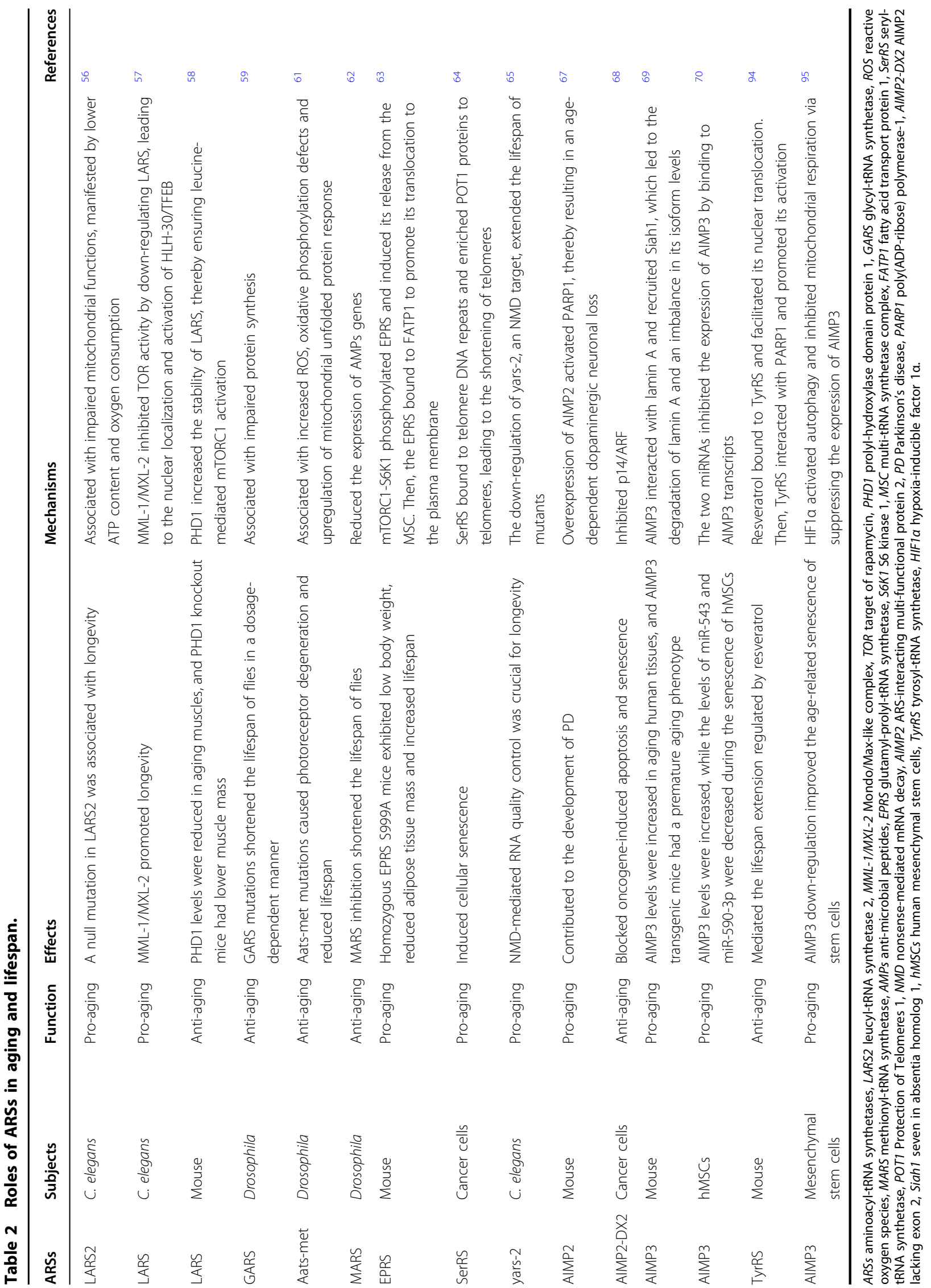



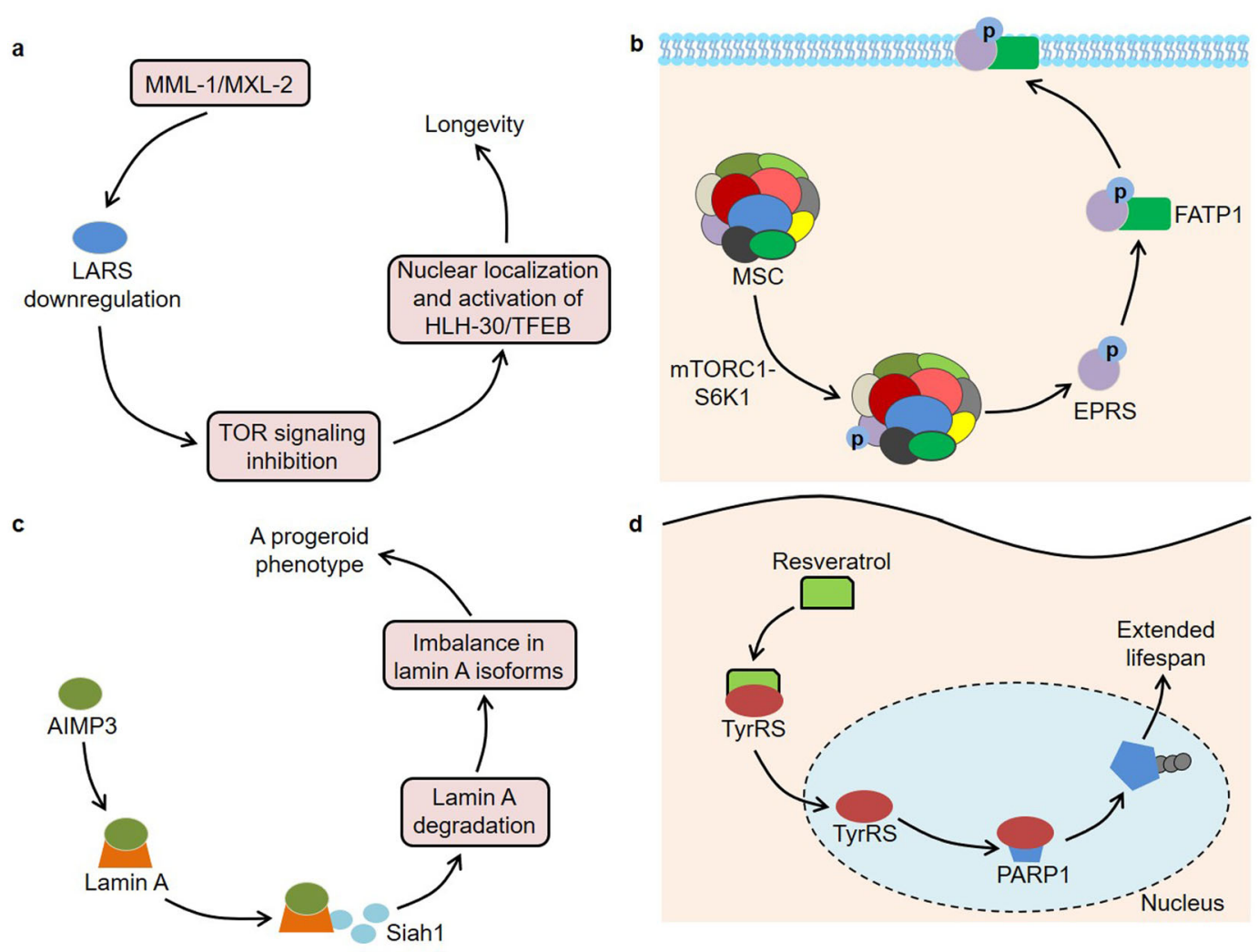

Fig. 3 The dysregulation of ARSs in aging and lifespan. a MML-1/MXL-2 inhibits TOR activity by down-regulating LARS, leading to the nuclear localization and activation of HLH-30/TFEB. $\mathbf{b}$ mTORC1-S6K1 phosphorylates EPRS and thus induces its release from the MSC. The phosphorylated EPRS binds to FATP1 to promote its translocation to the plasma membrane. c AIMP3 interacts with lamin A and recruits Siah1, which leads to the degradation of lamin A as well as an imbalance in its isoform levels. $\mathbf{d}$ Resveratrol binds to TyrRS and facilitates its nuclear translocation. Then, TyrRS interacts with PARP1 and promotes its activation by stimulating NAD ${ }^{+}$-dependent auto-poly-ADP-ribosylation.

leucyl-tRNA synthetase 2 (LARS2) was associated with longevity by screening 5690 genes of C. elegans ${ }^{56}$. The long-lived worms had impaired mitochondrial functions, manifested by lower ATP content and oxygen consumption. Furthermore, Mondo/Max-like complex (MML-1/ MXL-2) played an important role in the lifespan extension induced by germline removal ${ }^{57}$ (Fig. 3a). In this context, MML-1/MXL-2 inhibited TOR activity by downregulating LARS, leading to the nuclear localization and activation of HLH-30/TFEB. Another research observed that prolyl-hydroxylase domain protein 1 (PHD1) levels were reduced in aging muscles, and PHD1 knockout mice had lower muscle mass ${ }^{58}$. PHD1 increased the stability of LARS by interacting with it, thereby ensuring leucinemediated mTORC1 activation and maintaining muscle mass. These findings indicate that LARS participates in the biology of aging through different signaling pathways. Niehues et al. built a Drosophila model for Charcot-Marie-Tooth neuropathy by three mutations in glycyl-tRNA synthetase (GARS) ${ }^{59}$. Of note, the expression of these mutants, including GARS_E71G, GARS_G240R, and GARS_G526R, not only induced defects in neuronal morphology but also shortened the lifespan of flies in a dosage-dependent manner. In-depth research found that the mutant GARS proteins showed normal subcellular localization, but the overall protein synthesis in neurons was significantly reduced ${ }^{59}$. Interestingly, the heterozygous GARS $^{\mathrm{C} 201 \mathrm{R}}$ mice had a normal lifespan, while this mutation significantly rescued the shortened lifespan caused by the SOD $1^{\mathrm{G} 93 \mathrm{~A}}$ mutation ${ }^{60}$. Therefore, more studies are still needed to explore the roles of GARS in lifespan regulation.

The mutations in Aats-met, a homolog of human methionyl-tRNA synthetase 2 (MARS2), caused photoreceptor degeneration and reduced lifespan of flies, which was associated with the increased ROS, oxidative phosphorylation defects and upregulation of mitochondrial unfolded protein response ${ }^{61}$. Moreover, inhibition of MARS could shorten the lifespan of flies by reducing the expression of anti-microbial peptides genes ${ }^{62}$. Arif et al. discovered that glutamyl-prolyl-tRNA synthetase (EPRS) was a downstream effector of the mTORC1 and p70 ribosomal protein S6 kinase 1 (S6K1) axis, which was involved in the biological processes of obesity and aging ${ }^{63}$ 
(Fig. 3b). In terms of mechanism, mTORC1-S6K1 phosphorylated EPRS at Ser ${ }^{999}$ and thus induced its release from the MSC. Subsequently, the phosphorylated EPRS bound to fatty acid transport protein 1 (FATP1) to promote its translocation to the plasma membrane and longchain fatty acid uptake. Consistently, homozygous phospho-deficient EPRS S999A mice showed reduced adipose tissue, weight loss and longer lifespan, while replacement of the phospho-mimetic S999D allele restored the weight of s6k1-deficient mice to normal ${ }^{63}$. Furthermore, seryl-tRNA synthetase (SerRS) not only bound to telomere DNA repeats but also enriched Protection of Telomeres 1 (POT1) proteins to telomeres via direct interaction with POT1 in the nucleus ${ }^{64}$. The enrichment of POT1 led to the shortening of telomeres, thereby inhibiting the growth of HeLa cells by inducing cellular senescence. Intriguingly, the activity of nonsensemediated mRNA decay (NMD) decreased with the age of C. elegans, and NMD could contribute to the longevity in daf-2 mutant worms ${ }^{65}$. Further research has shown that downregulation of yars-2/tyrosyl-tRNA synthetase 2 (TyrRS2), an NMD target, effectively extends the lifespan of mutants, indicating that NMD-mediated RNA quality control plays an important role in organismal aging ${ }^{65}$.

Moreover, AIMPs, which mainly act as scaffolds in the MSC, is also associated with the aging process. It was reported that AIMP2 was a parkin substrate and contributed to the development of Parkinson's disease (PD) ${ }^{66}$. Overexpression of AIMP2 could activate poly(ADP-ribose) polymerase-1 (PARP1), thereby resulting in an agedependent dopaminergic neuronal loss in mice ${ }^{67}$. The PARP1 inhibitor AG014699 inhibited the degeneration of dopaminergic neurons in AIMP2 transgenic mice, indicating that PARP1 could be used as a target for PD treatment ${ }^{67}$. Notably, AIMP2-DX2, a splicing variant of AIMP2 lacking exon 2, was induced by oncogenes in human lung cancer cells and could block oncogene-induced apoptosis and senescence by inhibiting $\mathrm{p} 14 / \mathrm{ARF}^{68}$. In addition, endogenous AIMP3 levels increased in aging human tissues, and AIMP3 transgenic mice had an obvious premature aging phenotype, which was manifested as earlier cessation of weight gain, hair loss, reduced bone mineral deposits in female and bone thickness, lordokyphosis, as well as wrinkled skin with reduced adipocytes ${ }^{69}$ (Fig. 3c). Mechanistically, AIMP3 interacted with lamin A and recruited seven in absentia homolog 1 (Siah1), which led to the degradation of lamin A. The lamin A degradation would result in an imbalance in its isoform levels, thus inducing organismal aging. Analogously, Lee et al.$^{70}$ demonstrated that AIMP3 levels were increased, while the levels of miR543 and miR-590-3p were decreased during the senescence of human mesenchymal stem cells. These two microRNAs (miRNAs) could inhibit the expression of AIMP3 by binding to AIMP3 transcripts, thereby delaying cellular aging.

\section{tRNA derivatives in aging and lifespan}

Particularly, pre-tRNAs or mature tRNAs are cleaved into diverse subtypes of fragments under stress conditions, which are named tRNA derivatives ${ }^{71}$. Victoria et al. discovered that the circulating levels of $5^{\prime}$ tRNA halves derived from tRNA ${ }^{\mathrm{Cys}(\mathrm{GCA})}$ and $\mathrm{tRNA}^{\mathrm{Lys}(\mathrm{CTT})}$ were decreased, and those derived from tRNA ${ }^{\mathrm{His}(\mathrm{GTG})}$ and tRNA $^{\text {Asp(GTC) }}$ were increased with age in normal mice ${ }^{72}$. Importantly, the alterations in the levels of these $5^{\prime}$ tRNA halves were mitigated in the long-lived Ames dwarf mice. Likewise, another study found that the serum levels of certain specific $5^{\prime}$ tRNA halves changed significantly with age in mice, and their levels could be regulated by calorie restriction $^{73}$. These results suggest that tRNA fragments may play vital roles in the anti-aging effects of dwarfism or calorie restriction. In some cases, RNA molecules harboring a $2^{\prime}, 3^{\prime}$-cyclic phosphate (cP-RNAs) at the $3^{\prime}$ end are generated from endoribonuclease-mediated RNA cleavage $^{74}$. It was worth noting that $\mathrm{cP}$-RNAs, mainly from tRNAs, rRNAs, and mRNAs, were abundantly present in mouse tissues, and their levels declined in an agedependent manner ${ }^{75}$. Among them, the cP-RNAs derived from tRNAs were produced from the cleavage of anticodon loops and $3^{\prime}$-terminal CCA sequences. However, more studies are needed to explore the roles of tRNA derivatives in aging.

Of note, tRNA derivatives have been confirmed to be related to some age-related pathological processes, especially neurodegenerative diseases. Karaiskos et al. ${ }^{76}$ observed that the abundance of tRNA fragments in rat brains changed dynamically under the background of age. On the one hand, the levels of tRNA fragments derived from the $3^{\prime}$ end usually increased with age. On the other hand, the levels of tRNA fragments derived from the $5^{\prime}$ end were lower in the brains of middle-aged rats, while their levels were higher in the young and old rats. Interestingly, the potential targets of these fragments appeared to be enriched in neuronal functions and development, indicating that tRNA fragments might be involved in human aging and neurodegeneration ${ }^{76}$. Similarly, eight tRNA fragments were found to be differentially expressed in the brains of senescence-accelerated mouse prone 8 (SAMP8) mice, and these fragments seemed to regulate the brain function-associated pathways in a miRNA-like pattern $^{77}$. For example, AS-tDR-011775 could act on myelin-associated oligodendrocyte basic protein or parkin (PARK2), thus contributing to the development of brain aging-associated diseases ${ }^{77}$. Conspicuously, cleavage and polyadenylation factor I subunit 1 (CLP1) could facilitate the efficient generation of tRNA exons by maintaining the integrity of the tRNA splicing endonuclease complex, and CLP1 kinase-dead mice showed progressive loss of lower motor neurons ${ }^{78}$. At the mechanistic level, loss of CLP1 activity led to the accumulation of $5^{\prime}$ leader exon tRNA 
fragments derived from pre-tRNA ${ }^{\text {Tyr }}\left(5^{\prime}\right.$ Tyr-tRF $)$ and p53-mediated cell death. Further research found that the $5^{\prime}$ Tyr-tRF promoted the p53-dependent neuronal cell death by interacting with pyruvate kinase M2 (PKM2) ${ }^{79}$. In addition, Balaskas et al. discovered that various tRNA and tRNA fragments were differentially expressed between young and old equine chondrocytes ${ }^{80}$. Importantly, certain $5^{\prime}$ tiRNAs, such as tiRNA His-GTG and tiRNA Glu-TTC, were induced in both old equine chondrocytes and high-grade osteoarthritis cartilage, indicating that tRNA fragments might be involved in the development of age-related cartilage diseases ${ }^{80}$.

\section{Conclusion and future perspective}

Traditionally, tRNAs are considered as housekeeping molecules that mainly transport amino acids to the ribosome to participate in protein translation. After transcription, each tRNA needs to undergo a series of complex maturation processes to become functional ${ }^{81}$. In their metabolic process, defects in any step may cause various human diseases ${ }^{82-84}$. For example, the tRNAmodifying enzyme FTSJ1 was down-regulated in nonsmall cell lung cancer (NSCLC) tissues ${ }^{85}$. Importantly, FTSJ1 inhibited the growth of NSCLC cells by reducing the expression of DNA damage-regulated autophagy modulator 1. Furthermore, certain ARSs, including asparaginyl-tRNA synthetase, aspartyl-tRNA synthetase 2, and GARS, were associated with the development of neurological disorders ${ }^{86-88}$. As described above, tRNArelated metabolism, including tRNA transcription, tRNA molecules, tRNA modifications, tRNA aminoacylation, and tRNA derivatives, not only participates in cellular senescence but also plays a vital role in aging and longevity of organisms. In this context, studying tRNAs seems to provide new ideas for lifespan extension. However, the related molecular mechanism research is still in the initial stage, especially in the aspect of tRNA derivatives.

Indeed, some studies have begun to explore clinical transformations based on tRNA metabolism. Mutations in the human mitochondrial DNA (mtDNA) are implicated in age-associated disease phenotypes and aging ${ }^{89,90}$. Notably, specific mitoTALENs monomers for the tRNA $^{\text {Ala }}$ m.5024C $>$ T mutation could reduce the mutant mtDNA load and restore the tRNA ${ }^{\text {Ala }}$ levels in the muscle and heart of a mouse model of heteroplasmic mtDNA mutation $^{91}$. It was reported that the natural phenol resveratrol contributed to extending the lifespan of organisms ${ }^{92,93}$. Further research showed that resveratrol could bind to the active site of TyrRS and facilitate its nuclear translocation $^{94}$ (Fig. 3d). Then, TyrRS interacted with the C-domain of PARP1 and promoted its activation by stimulating $\mathrm{NAD}^{+}$-dependent auto-poly-ADPribosylation. Moreover, AIMP3 overexpression inhibited the functions of mesenchymal stem cells under hypoxic conditions, while the down-regulation of AIMP3 significantly improved the age-related senescence of stem cells ${ }^{95}$. Interestingly, hypoxia-inducible factor $1 \alpha$ (HIF1 $\alpha$ ) could activate autophagy and inhibit mitochondrial respiration via suppressing the expression of AIMP3, thereby delaying aging ${ }^{95}$. These findings provided a possible target for the regulation of aging. Another study found that the tRNA-derived fragments from the prefrontal cortex, cerebrospinal fluid and serum were differently expressed between PD patients and control samples, and they could distinguish PD from controls, indicating that tRNA fragments might serve as potential biomarkers for age-associated disease ${ }^{96}$. In conclusion, tRNA metabolism is closely related to aging and lifespan, and studying their relationship may become a hot topic in the future.

\section{Acknowledgements \\ The authors wish to acknowledge Chunsheng Zhu from the First Affiliated Hospital of Zhengzhou University, China for editing of English grammar and syntax of the paper. \\ Author details \\ ${ }^{1}$ Department of Chinese Medicine, The First Affiliated Hospital of Zhengzhou University, Zhengzhou 450000, China. '2Department of Pharmacy, The Second Xiangya Hospital, Central South University, Changsha 410011, China. \\ ${ }^{3}$ Institution of Clinical Pharmacy, Central South University, Changsha 410011, China}

\section{Author contributions}

Z.Z. and B.S. contributed to the drafting and revising of the article; M.B. and D.S $Y$. contributed to the conception and design; all authors approved the final version.

\section{Funding}

This project was supported by the National Natural Science Foundation of China (81900468).

\section{Ethical approval}

Not Applicable.

Conflict of interest

The authors declare that they have no conflict of interest.

\section{Publisher's note}

Springer Nature remains neutral with regard to jurisdictional claims in published maps and institutional affiliations.

Received: 26 February 2021 Revised: 11 May 2021 Accepted: 12 May 2021 Published online: 26 May 2021

\footnotetext{
References

1. Schaffer, A. E., Pinkard, O. \& Coller, J. M. tRNA metabolism and neurodevelopmental disorders. Annu. Rev. Genomics Hum. Genet. 20, 359-387 (2019).

2. Zhu, C., Sun, B., Nie, A. \& Zhou, Z. The tRNA-associated dysregulation in immune responses and immune diseases. Acta Physiol. 228, e13391 (2020).

3. Kwon, N. H., Fox, P. L. \& Kim, S. Aminoacyl-tRNA synthetases as therapeutic targets. Nat. Rev. Drug Discov. 18, 629-650 (2019).

4. Boskovic, A., Bing, X. Y., Kaymak, E. \& Rando, O. J. Control of noncoding RNA production and histone levels by a 5' tRNA fragment. Genes Dev. 34, 118-131 (2020).
} 
5. Mo, D. et al. A tRNA fragment, 5'-tiRNA ${ }^{\mathrm{Val}}$, suppresses the Wnt/ $\beta$-catenin signaling pathway by targeting FZD3 in breast cancer. Cancer Lett. 457, 60-73 (2019).

6. Deng, J. et al. Respiratory syncytial virus utilizes a tRNA fragment to suppress antiviral responses through a novel targeting mechanism. Mol. Ther. 23, 1622-1629 (2015)

7. Rosselló-Tortella, M. et al. Epigenetic loss of the transfer RNA-modifying enzyme TYW2 induces ribosome frameshifts in colon cancer. Proc. Natl Acad. Sci. USA 117, 20785-20793 (2020).

8. Blocquel, D. et al. CMT disease severity correlates with mutation-induced open conformation of histidyl-tRNA synthetase, not aminoacylation loss, in patient cells. Proc. Natl Acad. Sci. USA 116, 19440-19448 (2019).

9. Giannelou, A. et al. Aberrant tRNA processing causes an autoinflammatory syndrome responsive to TNF inhibitors. Ann. Rheum. Dis. 77, 612-619 (2018).

10. Zhou, Z., Sun, B., Huang, S., Jia, W. \& Yu, D. The tRNA-associated dysregulation in diabetes mellitus. Metabolism 94, 9-17 (2019).

11. Ferrucci, L. et al. Measuring biological aging in humans: a quest. Aging Cell 19, e13080 (2020)

12. Rusu, M. E. et al. Antioxidant effects of walnut (Juglans regia L.) kernel and walnut septum extract in a D-galactose-induced aging model and in naturally aged rats. Antioxidants 9, 424 (2020).

13. Chen, $L$. et al. 1,25-Dihydroxyvitamin $D$ exerts an antiaging role by activation of Nrf2-antioxidant signaling and inactivation of p16/p53-senescence signaling. Aging Cell 18, e12951 (2019).

14. Dluzen, D. F. et al. Extracellular RNA profiles with human age. Aging Cell 17, e12785 (2018)

15. Filer, D. et al. RNA polymerase III limits longevity downstream of TORC1. Nature 552, 263-267 (2017)

16. Lord, C. L., Ospovat, O. \& Wente, S. R. Nup100 regulates Saccharomyces cerevisiae replicative life span by mediating the nuclear export of specific tRNAs. RNA 23, 365-377 (2017).

17. Kaiser, F. J., Lüdecke, H. J. \& Weger, S. SUMOylation modulates transcriptional repression by TRPS1. Biol. Chem. 388, 381-390 (2007).

18. Singh, A. K. et al. SUMOylation of ROR-yt inhibits $\mathbb{L}-17$ expression and inflammation via HDAC2. Nat. Commun. 9, 4515 (2018).

19. Stankova, T., Piepkorn, L., Bayer, T. A., Jahn, O. \& Tirard, M. SUMO1-conjugation is altered during normal aging but not by increased amyloid burden. Aging Cell 17, e12760 (2018).

20. Rytinki, M. M. et al. Overexpression of SUMO perturbs the growth and development of Caenorhabditis elegans. Cell Mol. Life Sci. 68, 3219-3232 (2011).

21. Wang, X., Gerber, A., Chen, W. Y. \& Roeder, R. G. Functions of paralogous RNA polymerase III subunits POLR3G and POLR3GL in mouse development. Proc. Natl Acad. Sci. USA 117, 15702-15711 (2020).

22. Vannini, A. \& Cramer, P. Conservation between the RNA polymerase I, II, and III transcription initiation machineries. Mol. Cell 45, 439-446 (2012).

23. Neyret-Kahn, $H$. et al. Sumoylation at chromatin governs coordinated repression of a transcriptional program essential for cell growth and proliferation. Genome Res. 23, 1563-1579 (2013).

24. Wei, Y., Zhang, Y. J. \& Cai, Y. Growth or longevity: the TOR's decision on lifespan regulation. Biogerontology 14, 353-363 (2013).

25. Perić, M. et al. TORC1-mediated sensing of chaperone activity alters glucose metabolism and extends lifespan. Aging Cell 16, 994-1005 (2017).

26. Carroll, B. et al. Persistent mTORC1 signaling in cell senescence results from defects in amino acid and growth factor sensing. J. Cell Biol. 216, 1949-1957 (2017).

27. Rodríguez-López, M. et al. The GATA transcription factor Gaf1 represses tRNAs, inhibits growth, and extends chronological lifespan downstream of fission yeast TORC1. Cell Rep. 30, 3240-3249.e4 (2020).

28. Wei, Y., Tsang, C. K. \& Zheng, X. F. Mechanisms of regulation of RNA polymerase III-dependent transcription by TORC1. EMBO J. 28, 2220-2230 (2009).

29. Kantidakis, T., Ramsbottom, B. A., Birch, J. L., Dowding, S. N. \& White, R. J. mTOR associates with TFIIIC, is found at tRNA and 5 S rRNA genes, and targets their repressor Maf1. Proc. Natl Acad. Sci. USA 107, 11823-11828 (2010).

30. Zhang, S., Li, X., Wang, H. Y. \& Steven Zheng, X. F. Beyond regulation of pol III: Role of MAF1 in growth, metabolism, aging and cancer. Biochim. Biophys. Acta Gene Regul. Mech. 1861, 338-343 (2018).

31. Shetty, M. et al. Maf1-dependent transcriptional regulation of tRNAs prevents genomic instability and is associated with extended lifespan. Aging Cell 19, e13068 (2020).

32. Noguchi, C. et al. Maf1 limits RNA polymerase III-directed transcription to preserve genomic integrity and extend lifespan. Cell Cycle 20, 247-255 (2021).
33. Cai, Y. \& Wei, Y. H. Stress resistance and lifespan are increased in C. elegans but decreased in S. cerevisiae by mafr-1/maf1 deletion. Oncotarget 7, 10812-10826 (2016).

34. Bonhoure, N. et al. Loss of the RNA polymerase III repressor MAF1 confers obesity resistance. Genes Dev. 29, 934-947 (2015).

35. Sagi, D. et al. Tissue- and time-specific expression of otherwise identical tRNA genes. PLoS Genet. 12, e1006264 (2016).

36. McCormick, M. A. et al. A comprehensive analysis of replicative lifespan in 4,698 single-gene deletion strains uncovers conserved mechanisms of aging. Cell Metab. 22, 895-906 (2015).

37. Kimura, S., Srisuknimit, V. \& Waldor, M. K. Probing the diversity and regulation of tRNA modifications. Curr. Opin. Microbiol. 57, 41-48 (2020).

38. $\mathrm{Fu}, \mathrm{D}$. et al. Human AlkB homolog $\mathrm{ABH} 8 \mathrm{Is}$ a tRNA methyltransferase required for wobble uridine modification and DNA damage survival. Mol. Cell Biol. 30, 2449-2459 (2010)

39. Songe-Møller, L. et al. Mammalian ALKBH8 possesses tRNA methyltransferase activity required for the biogenesis of multiple wobble uridine modifications implicated in translational decoding. Mol. Cell Biol. 30, 1814-1827 (2010).

40. Leihne, $\mathrm{V}$. et al. Roles of Trm9- and ALKBH8-like proteins in the formation of modified wobble uridines in Arabidopsis tRNA. Nucleic Acids Res. 39, 7688-7701 (2011)

41. Lee, M. Y., Leonardi, A., Begley, T. J. \& Melendez, J. A. Loss of epitranscriptomic control of selenocysteine utilization engages senescence and mitochondrial reprogramming(浨). Redox Biol. 28, 101375 (2020).

42. Angelova, M. T. et al. tRNA 2'-O-methylation by a duo of TRM7/FTSJ1 proteins modulates small RNA silencing in Drosophila. Nucleic Acids Res. 48, 2050-2072 (2020).

43. Tischner, $\mathrm{C}$. et al. MTO1 mediates tissue specificity of OXPHOS defects via tRNA modification and translation optimization, which can be bypassed by dietary intervention. Hum. Mol. Genet. 24, 2247-2266 (2015).

44. Navarro-González, C. et al. Mutations in the Caenorhabditis elegans orthologs of human genes required for mitochondrial tRNA modification cause similar electron transport chain defects but different nuclear responses. PLoS Genet. 13, e1006921 (2017)

45. Fabrizio, P. et al. Genome-wide screen in Saccharomyces cerevisiae identifies vacuolar protein sorting, autophagy, biosynthetic, and tRNA methylation genes involved in life span regulation. PLoS Genet. 6, e1001024 (2010).

46. Begley, U. et al. A human tRNA methyltransferase 9-like protein prevents tumour growth by regulating LIN9 and HIF1-a. EMBO Mol. Med. 5, 366-383 (2013).

47. Van Haute, L. et al. NSUN2 introduces 5-methylcytosines in mammalian mitochondrial tRNAs. Nucleic Acids Res. 47, 8720-8733 (2019).

48. Tang, $H$. et al. NSun2 delays replicative senescence by repressing p27 (KIP1) translation and elevating CDK1 translation. Aging (Albany NY) 7, 1143-1158 (2015).

49. Cai, $X$. et al. RNA methyltransferase NSUN2 promotes stress-induced HUVEC senescence. Oncotarget 7, 19099-19110 (2016).

50. Goll, M. G. et al. Methylation of tRNAAsp by the DNA methyltransferase homolog Dnmt2. Science 311, 395-398 (2006).

51. Schaefer, M. et al. RNA methylation by Dnmt2 protects transfer RNAs against stress-induced cleavage. Genes Dev. 24, 1590-1595 (2010).

52. Lin, M. J., Tang, L. Y., Reddy, M. N. \& Shen, C. K. DNA methyltransferase gene dDnmt2 and longevity of Drosophila. J. Biol. Chem. 280, 861-864 (2005).

53. Lewinska, A., Adamczyk-Grochala, J., Kwasniewicz, E. \& Wnuk, M. Downregulation of methyltransferase Dnmt2 results in condition-dependent telomere shortening and senescence or apoptosis in mouse fibroblasts. J. Cell Physiol. 232, 3714-3726 (2017).

54. Lewinska, A. et al. Reduced levels of methyltransferase DNMT2 sensitize human fibroblasts to oxidative stress and DNA damage that is accompanied by changes in proliferation-related miRNA expression. Redox Biol. 14, 20-34 (2018).

55. Zhou, Z., Sun, B., Huang, S., Yu, D. \& Zhang, X. Roles of aminoacyl-tRNA synthetase-interacting multi-functional proteins in physiology and cancer. Cell Death Dis. 11, 579 (2020).

56. Lee, S. S. et al. A systematic RNAi screen identifies a critical role for mitochondria in C. elegans longevity. Nat. Genet. 33, 40-48 (2003).

57. Nakamura, S. et al. Mondo complexes regulate TFEB via TOR inhibition to promote longevity in response to gonadal signals. Nat. Commun. 7, 10944 (2016).

58. D'Hulst, G., Soro-Arnaiz, I., Masschelein, E. \& Veys, K. PHD1 controls muscle mTORC1 in a hydroxylation-independent manner by stabilizing leucyl tRNA synthetase. Nat. Commun. 11, 174 (2020). 
59. Niehues, S. et al. Impaired protein translation in Drosophila models for Charcot-Marie-Tooth neuropathy caused by mutant tRNA synthetases. Nat. Commun. 6, 7520 (2015).

60. Banks, G. T. et al. Mutant glycyl-tRNA synthetase (Gars) ameliorates SOD1 (G93A) motor neuron degeneration phenotype but has little affect on Loa dynein heavy chain mutant mice. PloS ONE 4, e6218 (2009).

61. Bayat, V. et al. Mutations in the mitochondrial methionyl-tRNA synthetase cause a neurodegenerative phenotype in flies and a recessive ataxia (ARSAL) in humans. PLoS Biol. 10, e1001288 (2012).

62. Suh, Y. S. et al. Methionyl-tRNA synthetase regulates lifespan in drosophila. Mol. Cells 43, 304-311 (2020).

63. Arif, A. et al. EPRS is a critical mTORC1-S6K1 effector that influences adiposity in mice. Nature 542, 357-361 (2017).

64. Li, Y. et al. Seryl tRNA synthetase cooperates with POT1 to regulate telomere length and cellular senescence. Signal Transduct. Target Ther. 4, 50 (2019).

65. Son, H. G. et al. RNA surveillance via nonsense-mediated mRNA decay is crucial for longevity in daf-2/insulin/IGF-1 mutant C. elegans. Nat. Commun. 8, 14749 (2017).

66. Corti, O. et al. The p38 subunit of the aminoacyl-tRNA synthetase complex is a Parkin substrate: linking protein biosynthesis and neurodegeneration. Hum. Mol. Genet. 12, 1427-1437 (2003).

67. Lee, Y. et al. Parthanatos mediates AIMP2-activated age-dependent dopaminergic neuronal loss. Nat. Neurosci. 16, 1392-1400 (2013).

68. Oh, A. Y. et al. Inhibiting DX2-p14/ARF interaction exerts antitumor effects in lung cancer and delays tumor progression. Cancer Res. 76, 4791-4804 (2016).

69. Oh, Y. S. et al. Downregulation of lamin A by tumor suppressor AIMP3/p18 leads to a progeroid phenotype in mice. Aging Cell 9, 810-822 (2010).

70. Lee, S. et al. miR-543 and miR-590-3p regulate human mesenchymal stem cell aging via direct targeting of AIMP3/p18. Age 36, 9724 (2014).

71. Sun, C. et al. Roles of tRNA-derived fragments in human cancers. Cancer Lett. 414, 16-25 (2018)

72. Victoria, B. et al. Circulating microRNA signature of genotype-by-age interactions in the long-lived Ames dwarf mouse. Aging Cell 14, 1055-1066 (2015).

73. Dhahbi, J. M. et al. 5' tRNA halves are present as abundant complexes in serum, concentrated in blood cells, and modulated by aging and calorie restriction. BMC Genomics 14, 298 (2013).

74. Shigematsu, M., Kawamura, T. \& Kirino, Y. Generation of 2',3'-cyclic phosphatecontaining RNAs as a hidden layer of the transcriptome. Front. Genet. 9, 562 (2018).

75. Shigematsu, M., Morichika, K., Kawamura, T., Honda, S. \& Kirino, Y. Genomewide identification of short 2',3'-cyclic phosphate-containing RNAs and their regulation in aging. PLOS Genet. 15, e1008469 (2019).

76. Karaiskos, S. \& Grigoriev, A. Dynamics of tRNA fragments and their targets in aging mammalian brain. F1000Res 5, ISCB Comm J-2758 (2016).

77. Zhang, S. et al. Identification of functional tRNA-derived fragments in senescence-accelerated mouse prone 8 brain. Aging (Albany NY) 11 10485-10498 (2019)
78. Hanada, T. et al. CLP1 links tRNA metabolism to progressive motor-neuron loss. Nature 495, 474-480 (2013).

79. Inoue, M. et al. Tyrosine pre-transfer RNA fragments are linked to p53dependent neuronal cell death via PKM2. Biochem. Biophys. Res. Commun. 525, 726-732 (2020).

80. Balaskas, P. et al. Small non-coding RNAome of ageing chondrocytes. Int J. Mol. Sci. 21, 5675 (2020).

81. Phizicky, E. M. \& Hopper, A. K. tRNA biology charges to the front. Genes Dev. 24, 1832-1860 (2010).

82. Ignatova, V. V. et al. METTL6 is a tRNA m(3)C methyltransferase that regulates pluripotency and tumor cell growth. Sci. Adv. 6, eaaz4551 (2020).

83. Auré, $K$. et al. Homoplasmic mitochondrial tRNA(Pro) mutation causing exercise-induced muscle swelling and fatigue. Neurol. Genet. 6, e480 (2020).

84. Liu, W. et al. Systematic analysis of tRNA-derived small RNAs discloses new therapeutic targets of caloric restriction in myocardial ischemic rats. Front. Cell Dev. Biol. 8, 568116 (2020).

85. He, Q. et al. FTSJ1 regulates tRNA 2'-O-methyladenosine modification and suppresses the malignancy of NSCLC via inhibiting DRAM1 expression. Cell Death Dis. 11, 348 (2020).

86. Wang, L. et al. Loss of NARS1 impairs progenitor proliferation in cortical brain organoids and leads to microcephaly. Nat. Commun. 11, 4038 (2020).

87. Rumyantseva, A., Motori, E. \& Trifunovic, A. DARS2 is indispensable for Purkinje cell survival and protects against cerebellar ataxia. Hum. Mol. Genet. 29 2845-2854 (2020).

88. Mo, Z. et al. Aberrant GlyRS-HDAC6 interaction linked to axonal transport deficits in Charcot-Marie-Tooth neuropathy. Nat. Commun. 9, 1007 (2018).

89. Kauppila, T. E. S., Kauppila, J. H. K. \& Larsson, N. G. Mammalian mitochondria and aging: an update. Cell Metab. 25, 57-71 (2017).

90. $\mathrm{Ma}, \mathrm{H}$. et al. Germline and somatic mtDNA mutations in mouse aging. PLoS ONE 13, e0201304 (2018).

91. Bacman, S. R. et al. MitoTALEN reduces mutant mtDNA load and restores tRNA (Ala) levels in a mouse model of heteroplasmic mtDNA mutation. Nat. Med. 24, 1696-1700 (2018).

92. Howitz, K. T. et al. Small molecule activators of sirtuins extend Saccharomyces cerevisiae lifespan. Nature 425, 191-196 (2003).

93. Yoon, D. S., Cha, D. S., Choi, Y., Lee, J. W. \& Lee, M. H. MPK-1/ERK is required for the full activity of resveratrol in extended lifespan and reproduction. Aging Cell 18, e12867 (2019)

94. Sajish, M. \& Schimmel, P. A human tRNA synthetase is a potent PARP1activating effector target for resveratrol. Nature 519, 370-373 (2015).

95. Kim, C., Park, J. M., Song, Y., Kim, S. \& Moon, J. HIFla-mediated AIMP3 suppression delays stem cell aging via the induction of autophagy. Aging Cell 18, e12909 (2019).

96. Magee, R., Londin, E. \& Rigoutsos, I. TRNA-derived fragments as sex-dependent circulating candidate biomarkers for Parkinson's disease. Parkinsonism Relat. Disord. 65, 203-209 (2019). 